\title{
HIERARCHICAL EVOLUTION IN POOR GROUPS OF GALAXIES
}

\author{
Ann I. Zabludoff ${ }^{1,2}$ and John S. Mulchaey ${ }^{1}$ \\ Accepted for publication in Astrophysical Journal Letters
}

\begin{abstract}
To examine the evidence for hierarchical evolution on mass scales of $\sim 10^{13}-10^{14} M_{\odot}$, we apply a statistic that measures correlations between galaxy velocity and projected position (Dressler \& Shectman 1988) to data for six poor groups of galaxies, HCG 42, HCG 62, NGC 533, NGC 2563, NGC 5129, and NGC 741. Each group has more than 30 identified members (Zabludoff \& Mulchaey 1998ab). The statistic is sensitive to clumps of galaxies on the sky whose mean velocity and velocity dispersion deviate from the kinematics of the group as a whole. The kinematics of galaxies within $\sim 0.1 h^{-1}$ Mpc of the group center do not deviate from the global values, supporting our earlier claim that the group cores are close to virialization or virialized. We detect significant substructure (at $\geq 99.9 \%$ confidence) in the two groups with the most confirmed members, HCG 62 and NGC 741, that is attributable mostly to a subgroup lying $\sim 0.3-0.4 h^{-1} \mathrm{Mpc}$ outside of the core. We conclude that at least some poor groups, like rich clusters, are evolving via the accretion of smaller structures from the field. With larger poor group surveys, the incidence of such accretion and the distribution of subgroup masses are potential constraints of cosmological models on mass scales of $\lesssim 10^{13}-10^{14} M_{\odot}$ and on physical scales of $\lesssim 0.5 h^{-1} \mathrm{Mpc}$.
\end{abstract}

Subject headings: galaxies: clustering — cosmology: large-scale structure of Universe

\footnotetext{
${ }^{1}$ Observatories of the Carnegie Institution of Washington, 813 Santa Barbara St., Pasadena, CA 91101, E-mail: mulchaey@pegasus.ociw.edu

${ }^{2} \mathrm{UCO} /$ Lick Observatory and Board of Astronomy and Astrophysics, University of California at Santa Cruz, Santa Cruz, CA, 95064, E-mail: aiz@ucolick.org
} 


\section{Introduction}

The evolution of structure on different mass scales is one of the outstanding issues in cosmology. For example, although galaxy clusters of $\sim 10^{15} M_{\odot}$ (including Virgo (Binggeli 1993; Bohringer et al. 1994), Coma (Mellier et al. 1988; Briel et al. 1992; White et al. 1993), and Abell 754 (Zabludoff \& Zaritsky 1995)) are clearly evolving from the accretion of smaller groups, it is uncertain whether poor groups of $\sim 10^{13}-10^{14} M_{\odot}$ also evolve hierarchically. There is some indirect evidence that the evolution of poor groups is similar to rich clusters; the galaxies and hot gas in poor groups follow the same relationships found among the X-ray temperature, X-ray luminosity, and galaxy velocity dispersion for rich clusters (Mulchaey \& Zabludoff 1998, hereafter MZ98). Historically, however, the number of known poor group members has been too small to examine individual groups for direct evidence of hierarchical evolution.

Multi-object spectroscopy now makes it possible to obtain "cluster-size" samples of galaxies in poor groups and to identify substructure, if it exists, in the same manner as for rich clusters. Substructure in clusters was not detected until the number of spectroscopically-confirmed cluster members exceeded $\sim 30-50$ galaxies. Recent poor group surveys have reached this membership level (Zabludoff \& Mulchaey 1998ab; hereafter ZM98a and b). The discovery of substructure in poor groups would provide new cosmological constraints by establishing that hierarchical evolution is occurring on mass scales of $\sim 10^{13}-10^{14} M_{\odot}$ and on physical scales of $\sim 0.5 h^{-1}$ Mpc. The detection of substructure would also support the picture in which at least some poor groups evolve as low-mass analogs to rich clusters.

In this Letter, we describe the results from applying a substructure statistic (Dressler \& Shectman 1988; hereafter DS88) to the six best sampled poor groups in ZM98ab, the most detailed spectroscopic survey of poor groups to date.

\section{The Group Sample}

In this analysis of substructure in poor groups, we consider six nearby systems $\left(3800<c z<7000 \mathrm{~km} \mathrm{~s}^{-1}\right)$ drawn from our spectroscopic survey of 12 opticallyidentified groups (ZM98a). We choose the six groups, HCG 42, HCG 62, NGC 533, NGC 741, NGC 2563, and NGC 5129, because each has more than 30 spectroscopicallyconfirmed members (see ZM98a for the details of the membership algorithm). The group members range in absolute magnitude from the three or four brightest $\left(M \lesssim M^{*}\right)$ galaxies identified in past surveys to the large population of dwarfs $\left(M \lesssim M^{*}+4\right)$ discovered in ZM98a. All six groups have X-ray halos extending out to radii of $\sim 200-300 \mathrm{kpc}$ and X-ray temperatures of $\sim 1 \mathrm{keV}$ (MZ98). In this Letter, we use the 
galaxy velocity data from ZM98a combined with new velocities for 51 additional group members (ZM98b). The number of known members within $\sim 0.5 h^{-1} \mathrm{Mpc}(\sim$ the virial radius) of each group center ranges from 35 to 63 galaxies.

\section{Results and Discussion}

To search for substructure in the six poor groups, we use the method applied by DS88 to rich clusters. This test identifies a fixed number of nearest neighbors on the sky around each galaxy, calculates the local mean velocity and velocity dispersion of the subsample, and compares these values with the mean velocity and velocity dispersion of the entire group. The kinematic deviations of the subsamples from the global values are summed. This sum is larger for a group with a kinematically distinct subgroup than for a similar group without substructure.

For each galaxy $i$, the deviation of its nearest projected neighbors from the kinematics of the group as a whole is defined as $\delta_{i} \equiv\left(n^{1 / 2} / \sigma_{r}\right)\left[\left(v_{l o c}-\bar{v}\right)^{2}+\left(\sigma_{r, l o c}-\right.\right.$ $\left.\left.\sigma_{r}\right)^{2}\right]^{1 / 2}$, where $\bar{v}$ is the mean velocity for the group, $\sigma_{r}$ is the group velocity dispersion, and $n$ is the number of nearest neighbors (including the galaxy) used to determine the local mean velocity $\bar{v}_{l o c}$ and local velocity dispersion $\sigma_{r, l o c}$. The total deviation for the group is defined as the sum of the local deviations, $\Delta \equiv \sum\left|\delta_{i}\right|$ for all $i \leq N_{\text {grp }}$, the number of group members. As pointed out in DS88, the $\Delta$ statistic is similar to the $\chi^{2}$ statistic, except that the $\delta_{i}$ 's are not squared before summation in order to reduce the contributions of the largest, rarest deviations. If the galaxy velocity distribution of the group is close to Gaussian, and the local variations are only random fluctuations, $\Delta \simeq N_{\text {grp }}$.

To calculate $\delta$, we choose $n=11$ (as in DS88). This choice allows robust determinations of $\bar{v}_{r, l o c}$ and $\sigma_{r, l o c}$. Silverman (1986) argues that using $n \sim N_{g r p}{ }^{1 / 2}$ nearest neighbors $(=6-8$ for these groups) maximizes the sensitivity of such a test to small scale structures while reducing its sensitivity to fluctuations within the Poisson noise (also see Bird 1994b). To check the robustness of the $n=11$ assumption, we compare the results below with those for $n=6$. The conclusions drawn from the $n=6$ and $n=11$ cases are the same.

Calibration of the $\Delta$ statistic for each group is required because 1) the $\delta_{i}$ 's are not statstically independent and 2) the velocity distribution may not be intrisically Gaussian even if there are no subgroups (e.g., the group members may follow predominantly circular or radial orbits). We determine the significance of the observed $\Delta$ by comparing it with the results of 1000 Monte Carlo trials in which galaxy velocities are drawn randomly from the observed distribution and assigned to galaxy positions. This scrambling technique effectively destroys any substructure (DS88). If the probability is low that a group without substructure has a $\Delta$ value at least as large as that observed, then we consider the substructure detection significant. 
In two groups, HCG 62 and NGC 741, the observed value of $\Delta$ is significant at the $\geq 99.9 \%$ confidence level. Such high $\Delta$ values might arise from substructure, but also could result from smooth variations in the group's velocity field (e.g., rotation or velocity shear (Malumuth et al. 1992)) and/or from a dependence of $\sigma_{r}$ on radius (Bird 1994b). ZM98a show that $\sigma_{r}$ is constant out to radii of $\sim 0.5 h^{-1} \mathrm{Mpc}$ in the combined velocity dispersion profile for the sample groups. To determine whether substructure is in fact responsible for the high $\Delta$ values in HCG 62 and NGC 741, we examine the local deviation $\delta$ for each group member. A concentration of large $\delta$ values on the sky indicates a kinematically distinct subgroup.

Figure 1 shows the projected spatial distribution of group members for each group (top panel). The second panel shows this distribution with the radii of the circles weighted by $e^{\delta}$ (as in DS88). Because each point is not statistically independent, a few very deviant galaxies can boost the $\delta$ values for a large number of nearby points. Therefore, a visual comparison with the Monte Carlo simulations is required to assess the significance of any structures. The third panel shows the results of the Monte Carlo trial (out of 1000) with the largest $\Delta$ value, or greatest total deviation. The results of the trial with the median $\Delta$ value are in the bottom panel.

The significance of the seven large clustered circles to the northeast of HCG 62 and five large clustered circles to the south of NGC 741 is high. In each case, the large $\delta$ values show that a subgroup not in equilibrium with the global group potential is the principal source of the significant $\Delta$ value. Each of the two subgroups lies a projected distance of $\sim 0.3-0.4 h^{-1} \mathrm{Mpc}$ outside of the group core.

On the other hand, the clustering of small circles within $\sim 0.1 h^{-1} \mathrm{Mpc}$ of all of the group centers indicates that the core mean velocity and velocity dispersion are similar to the global values for the group. This result suggests that the group cores are close to virialization or virialized and is consistent with the conclusions from our earlier studies of group dynamics (ZM98a, MZ98).

\section{Conclusions}

Of the six best sampled poor groups of galaxies (each has more than 30 known members), two have significant substructure. In each case, the substructure detection is due mostly to a subgroup lying $\sim 0.3-0.4 h^{-1} \mathrm{Mpc}$ outside of the core. It is worth noting that we detect substructure in the two groups, HCG 62 and NGC 741, with the most confirmed members and also that our substructure statistic (from DS88) is not sensitive to subgroups that are well-superposed on the sky (Bird 1993, 1994a). These two points suggest that the fraction of poor groups with substructure is higher than the $\sim 30 \%$ reported here. We conclude that poor groups, like rich clusters, evolve 
hierarchically and that some poor groups are still accreting smaller structures today 9 . In contrast, the cores of all of the poor groups (within $\sim 0.1 h^{-1} \mathrm{Mpc}$ ) appear to be relaxed, an observation consistent with our earlier claims that the cores are close to virialization or virialized (ZM98a, MZ98). Deep spectroscopic surveys of groups in a large group sample would place limits on both the incidence of substructure and the distribution of subgroup masses, providing a new test of cosmological models on mass scales of $\lesssim 10^{13}-10^{14} M_{\odot}$ and on physical scales of $\lesssim 0.5 h^{-1} \mathrm{Mpc}$.

We thank Dennis Zaritsky for his comments on the text. AIZ acknowledges support from the Carnegie and Dudley Observatories, the AAS, NSF grant AST-95-29259, and NASA grant HF-01087.01-96A. JM acknowledges support provided by NASA grant NAG 5-2831 and NAG 5-3529.

\footnotetext{
${ }^{3}$ Even the Local Group, which is less massive than these groups $\left(\sim 4 \times 10^{12} M_{\odot}\right.$ [Zaritsky et al. 1989] versus $\sim 10^{13}-10^{14} M_{\odot}[\mathrm{ZM} 98 \mathrm{a}]$ ), is forming from the collapse of the Milky Way and M 31 subgroups (Kahn \& Woltjer 1959). There is also some evidence that AWM/MKW poor clusters, which are typically $\sim 2-4$ times more massive than these groups, have bound clumps at much larger radii ( $\gtrsim 1-2 h^{-1} \mathrm{Mpc}$; Beers et al. 1995).
} 


\section{References}

Beers, T.C., Kriessler, J.R., Bird, C.M., \& Huchra, J.P. 1995, AJ, 109, 874

Binggeli, B. 1993, A \& A, 98, 275

Bird, C. 1993, Ph.D. Thesis, University of Minnesota and Michigan State University.

Bird, C. 1994a, AJ, 107, 1637

Bird, C. 1994b, AJ, 422, 480

Bohringer, H., Briel, U.G., Schwarz, R.A., Voges, W., Hartner, G. \& Trumper, J. 1994, Nature, 368, 828

Briel, U.G., Henry, J.P., \& Bohringer, H. 1992, A \& A, 259, L31

Dressler, A. \& Shectman, S. 1988, AJ, 95, 985 (DS88)

Kahn, F.D. \& Woltjer, L. 1959, ApJ, 130, 705

Malumuth, E.M., Kriss, G.A., Dix, W.V.D., Ferguson, H.C., \& Ritchie, C. 1992, AJ, 104, 495

Mellier, Y., Mathez, G., Mazure, A., Chauvineau, B., Proust, D. 1998, A \& A, 199, 67

Mulchaey, J. S. \& Zabludoff, A.I. 1998, ApJ, in press. (MZ98)

Silverman, B. 1986, Density Estimation for Statistics and Data Analysis (London:

Chapman and Hall)

White, S.D.M., Briel, U., \& Henry, J.P. 1993, M.N.R.A.S., 261, L8

Zabludoff, A.I. \& Zaritsky, D. 1995, ApJL, 447, L21

Zabludoff, A.I. \& Mulchaey, J.S. 1998a, ApJ, in press. (ZM98a)

Zabludoff, A.I. \& Mulchaey, J.S. 1998b, in prep. (ZM98b)

Zaritsky, D., Olszewski, E.W., Schommer, R.A., Peterson, R.C., \& Aaronson, M. 1989, ApJ, 345, 759 


\section{Figure Captions}

Figure 1: For each group, the top panel shows the distribution of group members on the sky. There are 35 galaxies in HCG 42, 63 in HCG 62, 36 in NGC 533, 41 in NGC 741, 44 in NGC 2563, and 38 in NGC 5129. The dashed line in the NGC 741 and NGC 5129 fields is the survey boundary (see ZM98a). The second panel is the same as the top panel, except that the radii of the circles are scaled by $e^{\delta}$, where $\delta$ is the deviation of the nearest neighbors from the kinematics of the group as a whole. The third panel shows the results of the Monte Carlo trial (out of 1000) with the largest value of the Dressler-Shectman (DS88) statistic $\Delta$. The bottom panel is the trial with the median value of $\Delta$. The scale bar below the bottom panel is $0.5 h^{-1} \mathrm{Mpc}$. 


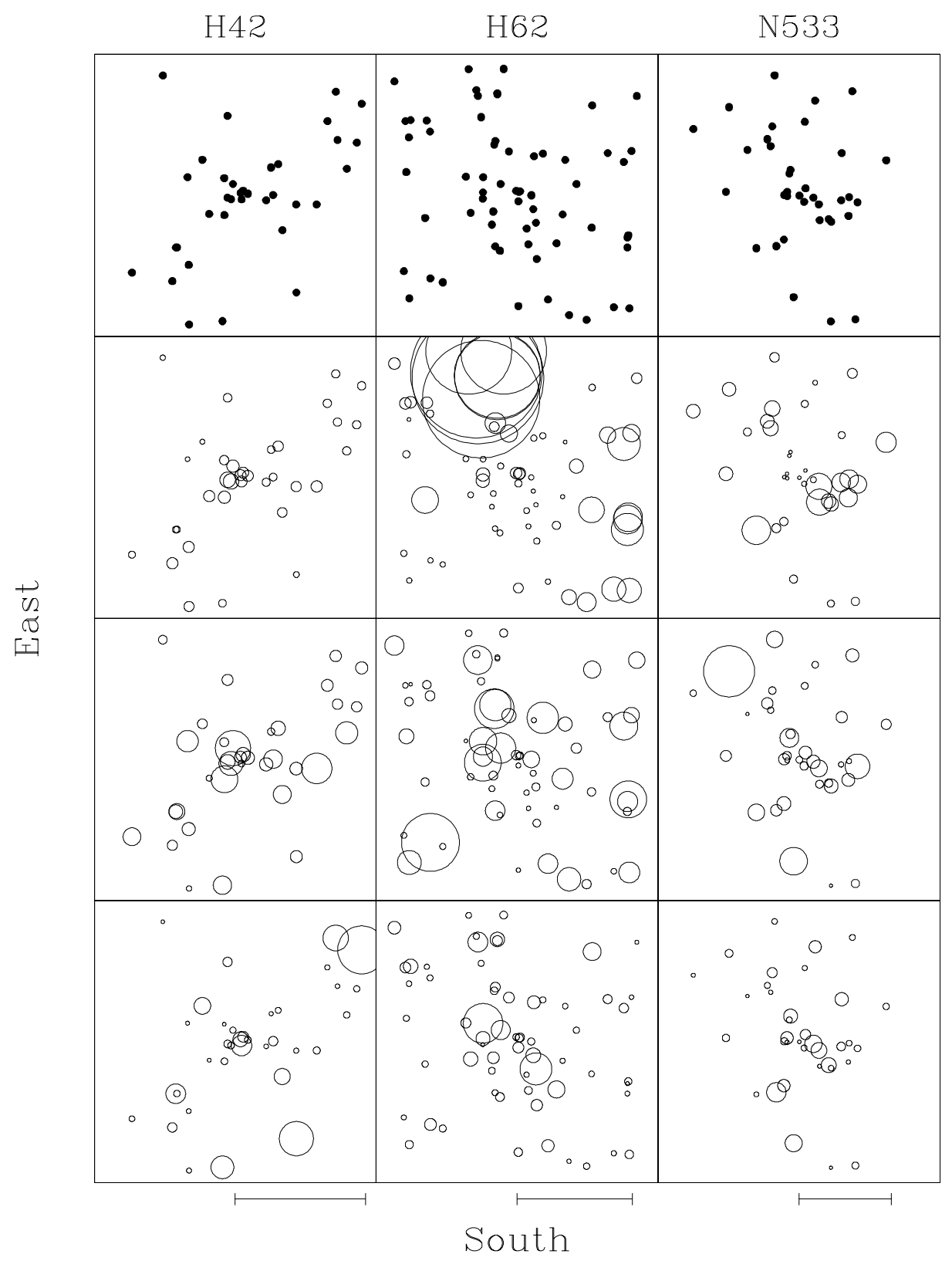

Fig. 1.- 


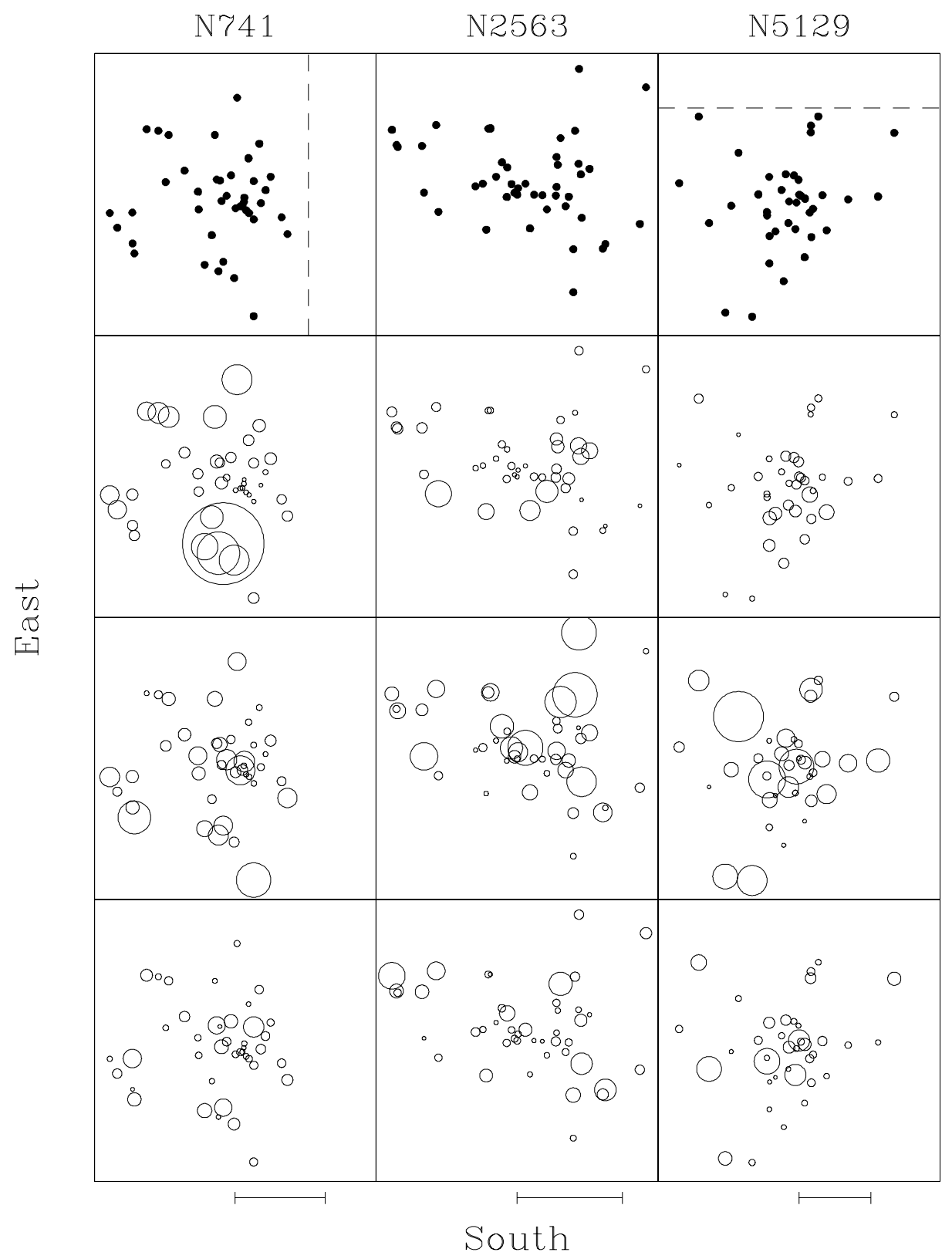

Fig. 1.- cont. 